\title{
Lesiones fatales en adolescentes, Casanare-Colombia 2011-2013
}

\author{
Claudia Yaneth Rincón Acevedo ${ }^{1}$, Liliana Patricia Zuleta Dueñas ${ }^{2}$, Oneida Castañeda-Porras ${ }^{3}$
}

Oficina de Vigilancia en Salud Pública, Secretaría de Salud de Casanare, Colombia

1 Bacterióloga y Laboratorista clínico, Esp. Epidemiología . Profesional de apoyo Grupo de Vigilancia, Secretaría de Salud de Casanare, evitalescasanare@gmail. com

2 Bacterióloga, Esp. Epidemiología, Epidemióloga de Campo Profesional universitario, Referente Grupo de Vigilancia, Secretaría de Salud de Casanare, vspcasanare@gmail.com

3 Psicóloga, Epidemióloga de Campo, Maestrante en Salud Pública, Profesional de apoyo Grupo de Vigilancia, Secretaría de Salud de Casanare, ocastaneda@ smc-as.com

Correo electrónico: evitalescasanare@gmail.com

Fecha de Recepción: 26/11/2015

Fecha de Evaluación: 2/02/2016

Fecha de Solicitud de Correcciones: 18/2/2016

Fecha de Aceptación: 24/02/2016

\section{Resumen.}

Antecedentes. Según la Organización Mundial de la Salud, las lesiones fatales son una de las principales causas de muerte y discapacidad en menores de 18 años. El objetivo del estudio fue describir su comportamiento en los adolescentes del departamento de Casanare, durante el período 2011-2013.

Materiales y métodos. Estudio descriptivo, a partir de las bases de datos de estadísticas vitales, defunciones no fetales y defunciones por causa externa del Departamento Administrativo Nacional de Estadística (DANE). El análisis de los datos se realizó en la hoja de cálculo de Excel 2010 de Microsoft Office ${ }^{\varpi}$. Se analizaron las tasas de mortalidad específica y ajustada por 100.000 habitantes por año, sexo, grupo etario y municipio; y los años de vida potencial perdidos (AVPP) por cada causa según la lista OPS-6/67 CIE-10.

Resultados. En el período hubo un total de 99 defunciones, en 2011 (23; 23,2\%), $2012(36 ; 36,4 \%)$ y $2013(40 ; 40,4 \%)$. Tasa de mortalidad específica fue de 138,2 y ajustada de 236,4 por 100.000 habitantes, en el grupo de 1014 años $(52,8 ; 45,4)$ y de $15-19(224,3 ; 189,9)$, en hombres $(220,0 ; 187,7)$ y mujeres $(54,1 ; 46,2)$. Total de AVPP 5.940, por accidentes de transporte 2.160, agresiones/homicidios 1.440 , suicidios 840 , intención no determinada 780 , ahogamiento 420, intervención legal/operaciones de guerra y otros accidentes 120 respectivamente, armas de fuego 60; índice de AVPP 83,1 por 1.000 habitantes.

Conclusiones. La primera causa de mortalidad por lesiones fatales fue por accidentes de transporte, situación diferente a la del país en la que los homicidios ocupan el primer lugar. Es necesario congregar esfuerzos desde todos los sectores para reducir la mortalidad de todos los niños.

Palabras clave. Lesiones, mortalidad, violencia, años potenciales de vida perdidos, adolescente, Colombia.
Fatal injuries in adolescents at Casanare - Colombia; 2011-2013

\section{Abstract.}

Background. According to World Health Organization, fatal injuries are among the main causes of death and disability in people less than $<18 \mathrm{yr}$. The purpose of this study is to describe injuries' profile in adolescents at department of Casanare during 2011-2013.

Methods.

Descriptive study from the non-fetal and external cause fatalities in the vital statistics database of the Administrative Department of National Statistics

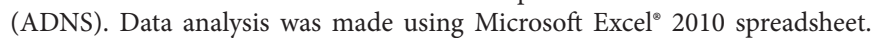
Specific and adjusted mortality rates per 100.000 inhabitants per year, agegroup and town, as well as years of potential life lost (YPLL) were analyzed for each cause according to OPS-6/67 CIE-10.

Results. During 2011-2013 there were 99 deaths: 2011, 23 (23,2\%); 2012, $36(36,4 \%) ; 2013,40(40,4 \%)$. Mortality rate was 138.2 and adjusted 236,4 per 100.000 inhabitants; by age-group: $10-14$ yr (52.8; 45,4/100.000); 15-19 $(224.3 ; 189,9 / 100.000)$; by sex: men $(220,0 ; 187,7 / 100.000)$; women $(54.1$; $46,2 / 100.000)$. YPLL were 5.940 ; by traffic accidents, 2.160; aggression/ homicide, 1.440; suicide, 840; undetermined, 780; drowning, 420; legal interventions/war operations and other related, 120; firearms, 60. Indices AVPP 83.1/1.000.

Conclusions. First cause of mortality due to fatal injuries was related to traffic accidents, a different picture from that of the whole nation, where homicides are the first issue. It is necessary to join efforts from all socio-economic sectors to decrease children's mortality.

Key words. Injury, Mortality, Violence, Potential Years of Life Lost, Adolescent, Colombia

1.

\section{Introducción}

Las lesiones fatales son todas aquellas que por su gravedad generan la causa de muerte de una persona y se dividen en homicidios, suicidios, accidentales, muertes violentas por accidente de transporte y muertes violentas indeterminadas (que incluyen incendios, quemaduras, ahogamientos, caídas, accidentes causados por máquinas, por instrumentos cortantes o punzantes, por arma de fuego, envenenamiento y exposición a sustancias nocivas) $(1,2)$. Según la Organización Mundial de la Salud (OMS), todos los días, la vida de más de 15.000 personas es interrumpida como consecuencia de una lesión, y cada año aproximadamente 5,8 millones de personas mueren por esta causa, lo que representa el $10 \%$ del total de las muertes mundiales (3).

Las estimaciones de la OMS, en el mundo, respecto a la proporción de lesiones fatales de acuerdo con el mecanismo de la lesión, la tasa de mortalidad por 100.000 habitantes por región (4) y la tasa de homicidios en adolescentes(5); se presenta en el cuadro. 
Cuadro 1. Estimaciones de la OMS respecto a lesiones fatales según el mecanismo de la lesión y las tasas por 100.000 habitantes de mortalidad y homicidios

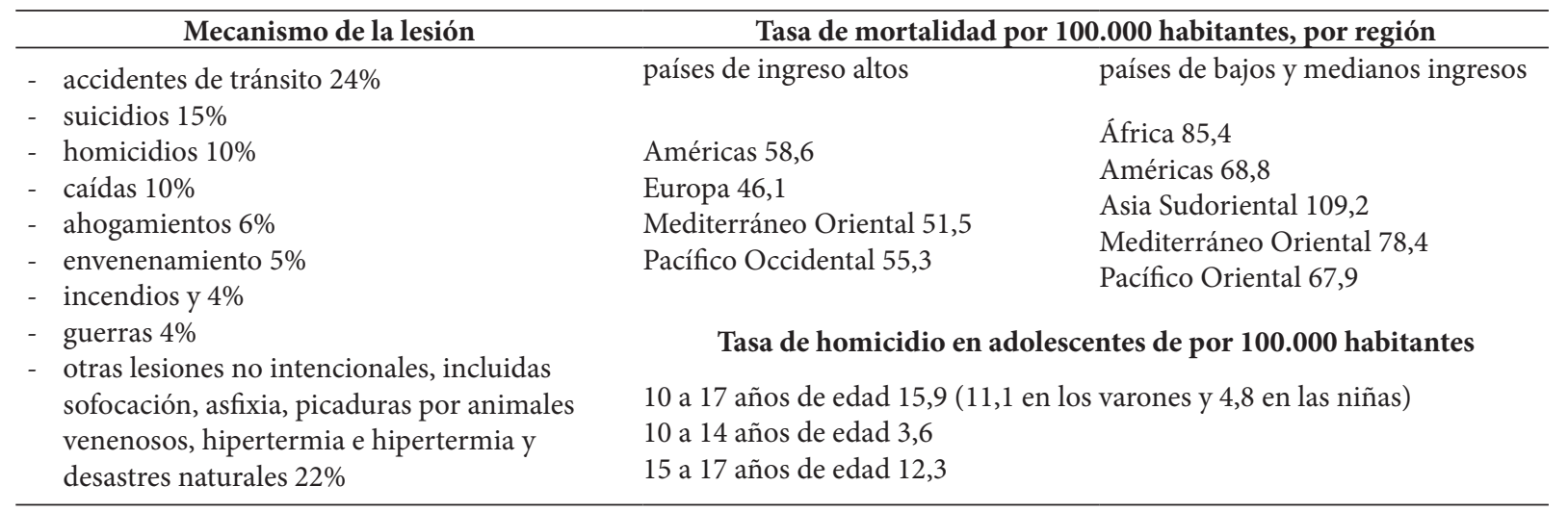

Las lesiones no intencionales representan casi el $90 \%$ de los casos de muerte en niños y jóvenes menores de 18 años de edad y son la principal causa de muerte en los menores entre 10 a 19 años de edad (6); en 2004, la distribución de las defunciones debidas a las lesiones en menores de 17 años, por traumatismos causados por el tránsito fue de $22,3 \%$, por ahogamientos de $16,8 \%$, quemaduras por fuego de $9,7 \%$, homicidios de $5,8 \%$, lesiones autoinfligidas de $4,4 \%$, caídas de $4,2 \%$, intoxicaciones de $3,8 \%$, guerra de $2,3 \%$ y otras lesiones no intencionales $31,1 \%(6)$.

En Colombia, para el período 2005-2012 dentro de la mortalidad general por grandes causas, las causas externas constituyeron la cuarta causa de muerte con un $17,1 \%$ del total de la mortalidad en el periodo, $\mathrm{y}$ aunque no fueron la primera causa de deceso, fueron las que mayor número de años potenciales de vida perdidos (AVPP) generaron. En efecto, durante el periodo aportaron el 30,8\% (11.968.667) de todos los AVPP y a pesar de experimentar una reducción del $21 \%$, para 2012 se generaron 2.880,81 AVPP por cada 100.0000 habitantes. Los homicidios provocaron el $6,5 \%$ del total de muertes por causas externas, los accidentes de transporte generaron el $18,8 \%$ y se ubicaron en el segundo lugar de frecuencia. En tercer lugar, los eventos de intención no determinada causaron muertes entre 4,3 y 5,04 por cada 100.000 habitantes durante el período y las tasas ajustadas de lesiones autoinfligidas (suicidios) pasaron de 5,1 a 4,6 (7). Durante el 2012, según el Instituto Colombiano de Medicina Legal y Ciencias Forenses (ICML-CF), el Sistema Médico Forense Colombiano practicó 28.496 necropsias médico-legales cuya forma de muerte fue violenta, clasificadas en homicidios 55\%, suicidios 7\%, muertes en accidentes de tránsito $22 \%$, muertes accidentales $10 \%$ y violentas indeterminadas $6 \%(8-14)$.

En el departamento de Casanare, en el 2000, la proporción de muertes por lesiones fue de $33,7 \%$, en hombres $41,9 \%$ y mujeres $20,5 \%$ mientras que la del país fue de $21,7 \%$, hombres $31,7 \%$ y mujeres $7,9 \%$; entre las primeras veinte causas de muerte, la tasa estandarizada por mil habitantes en hombres por agresiones fue 167,1 (primera causa), accidentes de tránsito 55,1 , otras lesiones no intencionales 28,8 , lesiones autoinfligidas 19,5, ahogamiento y sumersión accidental 13,9; y en mujeres por agresiones fue de 39,6, accidentes de tránsito 20,5 y otras lesiones no intencionales 17,8 , y en hombres (15). El homicidio fue la causa del $23 \%$ de las defunciones, cifra que descendió al 11,0\% en 2006 (16). Para el período 2005-2010, entre las diez primeras causas de mortalidad, los accidentes de transporte terrestre inclusive secuelas aportaron en promedio el 3,1\% de los fallecimientos; $y$, entre las lesiones fatales en menores de 17 años, las primeras cinco causas de muerte fueron por accidentes de transporte $28,8 \%$, ahogamiento y sumersión $19,2 \%$, homicidios $15,7 \%$, otros accidentes $13,2 \%$ y eventos con intención no determinada 11,1\% (12). Para el período 1998-2011, la tasa de mortalidad por lesiones de causa externa fue 125,22 por 100.00 habitantes, con el noveno lugar entre los departamentos que presentaron tasas de mortalidad superiores al promedio nacional de 93,61 (17); en 2012, entre los departamentos que tuvieron tasas ajustadas de mortalidad por causas externas significativamente más altas que la nacional de 66,3, el departamento de Casanare ocupó el sexto lugar con una tasa de 99,7 por 100.000 habitantes (7); y, en 2013, los cinco departamentos que reportaron las mayores tasas de muertes por cada 100.000 habitantes fueron Arauca 104,86, Valle del Cauca (04,17, Casanare 87,49, Putumayo 83,67 y Meta 75,25 (18)

Desde el enfoque del curso de vida uno de los períodos críticos es la transición de la adolescencia a la adultez, en los cuales la exposición a ciertos factores ambientales puede ser más nociva para la salud y afectar más el potencial de salud a largo plazo que en otros momentos de la vida $(19,20)$; entre esos factores, las lesiones son una de las principales causas de muerte y discapacidad en los menores de 18 años; y, dado que, aun cuando, las lesiones no son inevitables; pueden prevenirse y controlarse (6); por tanto, el objetivo del estudio fue describir el comportamiento de las lesiones fatales en los adolescentes del departamento de Casanare, durante el período 2011-2013, con el propósito de contribuir con datos actualizados que permitan fortalecer e implementar las acciones de prevención y control orientadas a la reducción de la mortalidad en este grupo poblacional.

\section{Materiales y métodos}

Estudio descriptivo, transversal, retrospectivo de fuente secundaria, a partir de las bases de datos de estadísticas vitales, defunciones no fetales y defunciones por causa externa del Departamento Administrativo Nacional de Estadística (DANE), ocurridas en el departamento de Casanare durante el período 2011-2013. Las causas de mortalidad se agruparon según la lista OPS-6/67 (21) y la Clasificación Internacional de Enfermedades (CIE-10) (22). El análisis de los datos se llevó a cabo en la hoja de cálculo de Excel 2010 de Microsoft Office ${ }^{\oplus}$.

Se analizaron las tasas de mortalidad específica por 100.000 habitantes por año, sexo, grupo etario de 10 a 14 años -adolescencia temprana- $y$ 15 a 19 años -adolescencia tardía- (23) y municipio, agrupadas por todas las causas externas: accidentes de transporte, inclusive secuelas; ahogamiento y sumersión accidentales; otros accidentes inclusive secuelas; lesiones autoinfligidas intencionales (suicidios); agresiones (homicidios); intervención legal y operaciones de guerra; y, eventos de intención no determinada. Los datos de población se obtuvieron 
de las proyecciones de población del Departamento Nacional de Estadística (DANE) que toma como base los resultados ajustados de población del Censo 2005 y la conciliación censal 1985-2005. Para hacer comparables las estimaciones de mortalidad se realizó el ajuste de tasas de mortalidad por el método indirecto, para cada una de las lesiones por grupo de edad y sexo con base en los valores de población estándar mundial de la OMS (24): en el grupo etario de 10 a 14 años de 85.970 habitantes, de los cuales, hombres y mujeres 42.985 respectivamente, en el grupo de 15 a 19 años de 84.670 habitantes, de ellos, hombres y mujeres 42.335 cada uno; y para el grupo de 10 a 19 años de 170.640 habitantes, por el número de defunciones por 100.000 habitantes.

Se calcularon los años de vida potencial perdidos por lesiones fatales por cada causa de muerte, por grupo quinquenal de edad y sexo, sumando los productos del número de muertes de dada edad por la diferencia entre esta edad y una edad límite, se tomó la esperanza de vida calculada por la OMS para Colombia en 2010, de 74 años (hombres 74,3 y mujeres 78,5). Para el análisis del indicador se construyó una tabla en la hoja de cálculo de Excel de Microsoft Office ${ }^{\oplus}$ con la siguiente información: intervalo de edad ( 10 a 14, 15 a 19 y 10 a 19), punto medio del intervalo $(12,5 ; 17,5$ y 14,0$)$ respectivamente, factor de ponderación o expectativa de vida $(61,5 ; 56,5$ y 60,0$)$ y el número de muertes en el período a estudio. Se calculó el índice de AVPP multiplicando los años de vida potencialmente perdidos de cada quinquenio por el total de la población de cada grupo etario multiplicada por un factor (1.000).

\section{Consideraciones éticas}

El estudio se clasifica como investigación sin riesgo, según la Resolución 008430 de 1993 del Ministerio de Salud (25), en cumplimiento de las actividades y obligaciones contempladas para la vigilancia epidemiológica en el Decreto 3518 de 2006 (26).

\section{Resultados}

En el período a estudio hubo un total de 99 defunciones, de las cuales, en 2011 (23; 23,2\%), en $2012(36 ; 36,4 \%)$ y en $2013(40 ; 40,4 \%)$ con un incremento de $56,5 \%$ entre 2011 y 2012, de $11,1 \%$ entre 2012 y 2013 y de $73,9 \%$ entre 2011 y 2013; por grupo etario, de 10 a 14 años (19; $19,2 \%)$ y de 15 a 19 años (80; 80,8\%); según el sexo, hombres $80(80,8 \%)$ y mujeres $19(19,2 \%)$; la razón hombre mujer fue de 4:1; distribuidas de acuerdo con el tipo de lesión, por accidentes de transporte terrestre 36 (36,4\%); agresiones/homicidios 27 (27,3\%); lesiones autoinfligidas intencionales/suicidios 14 (14,1\%); eventos de intención no determinada 13 (13,1\%); ahogamiento y sumersión accidentales $7(7,1 \%)$; otros accidentes incluido secuelas e intervención legal y operaciones de guerra $2(2,0 \%)$ respectivamente; $y$, accidentes causados por disparo de armas de fuego $1(1,0 \%)$. 
Figura 1. Tasa específica de mortalidad por lesiones fatales según edad y sexo, adolescentes, Casanare, 2011-2013

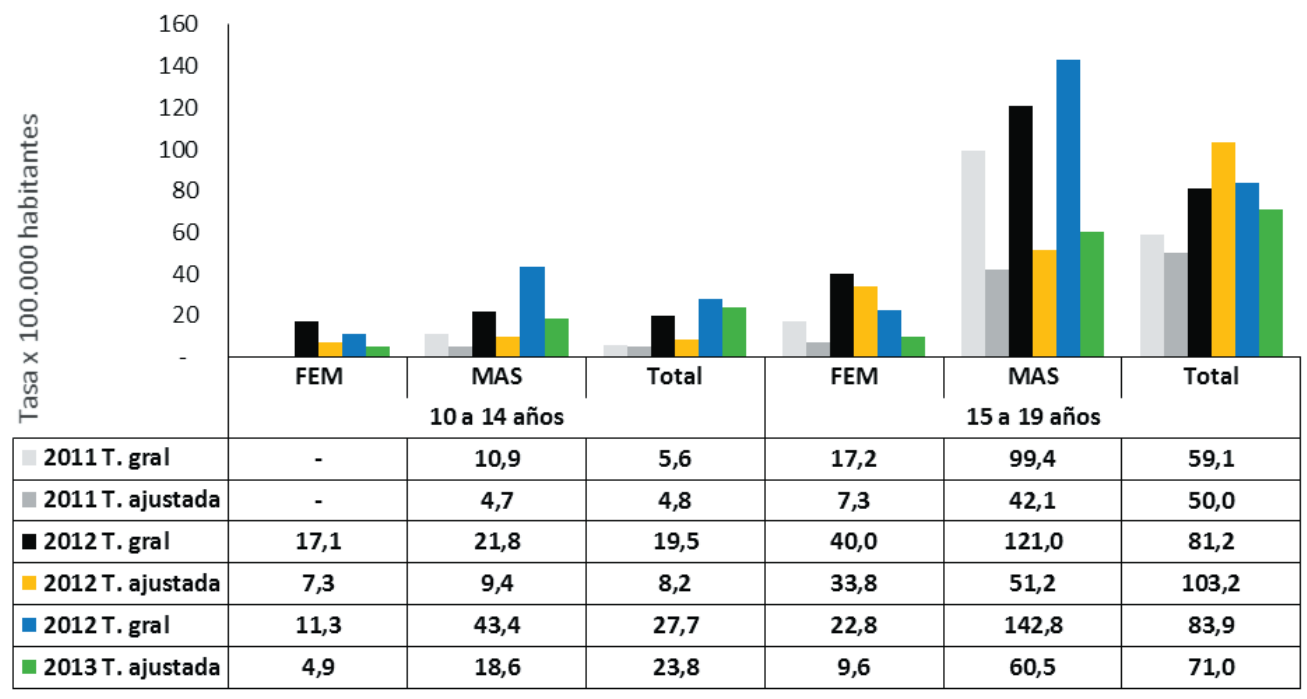

Figura 2. Tasas específica de mortalidad de lesiones fatales según diagnóstico 6/67 CIE-10 de OPS/OMS, por sexo y grupo etario, adolescentes, Casanare-Colombia, 2011-2013

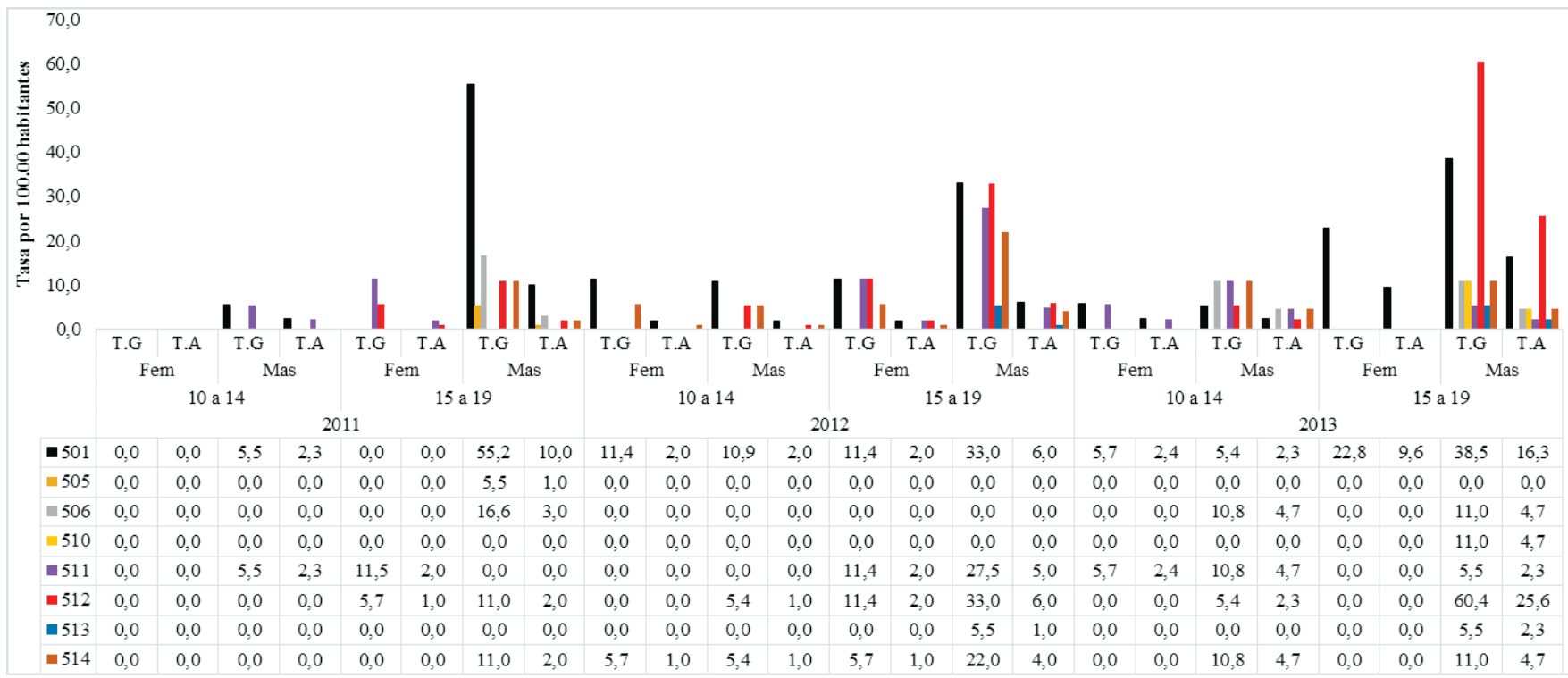

\begin{tabular}{ll}
\hline T.G Tasa general & T.A. Tasa ajustada \\
\hline 501 Accidentes de transporte terrestre, inclusive secuelas & $\begin{array}{l}511 \text { Lesiones autoinfligidas intencionales } \\
\text { (suicidios), , inclusive secuelas }\end{array}$ \\
505 Accidentes causados por disparo de armas de fuego & $\begin{array}{l}512 \text { Agresiones (homicidios), inclusive secuelas } \\
513 \text { Intervención legal y operaciones de guerra, } \\
\text { inclusive secuelas }\end{array}$ \\
506 Ahogamiento y Sumersión Accidentales & $\begin{array}{l}514 \text { Eventos de intención no determinada, } \\
\text { inclusive secuelas }\end{array}$ \\
\hline
\end{tabular}


Figura 3. Tasa específica de mortalidad por lesiones fatales según tipo de lesión y grupo etario, adolescentes, Casanare, 2011-2013

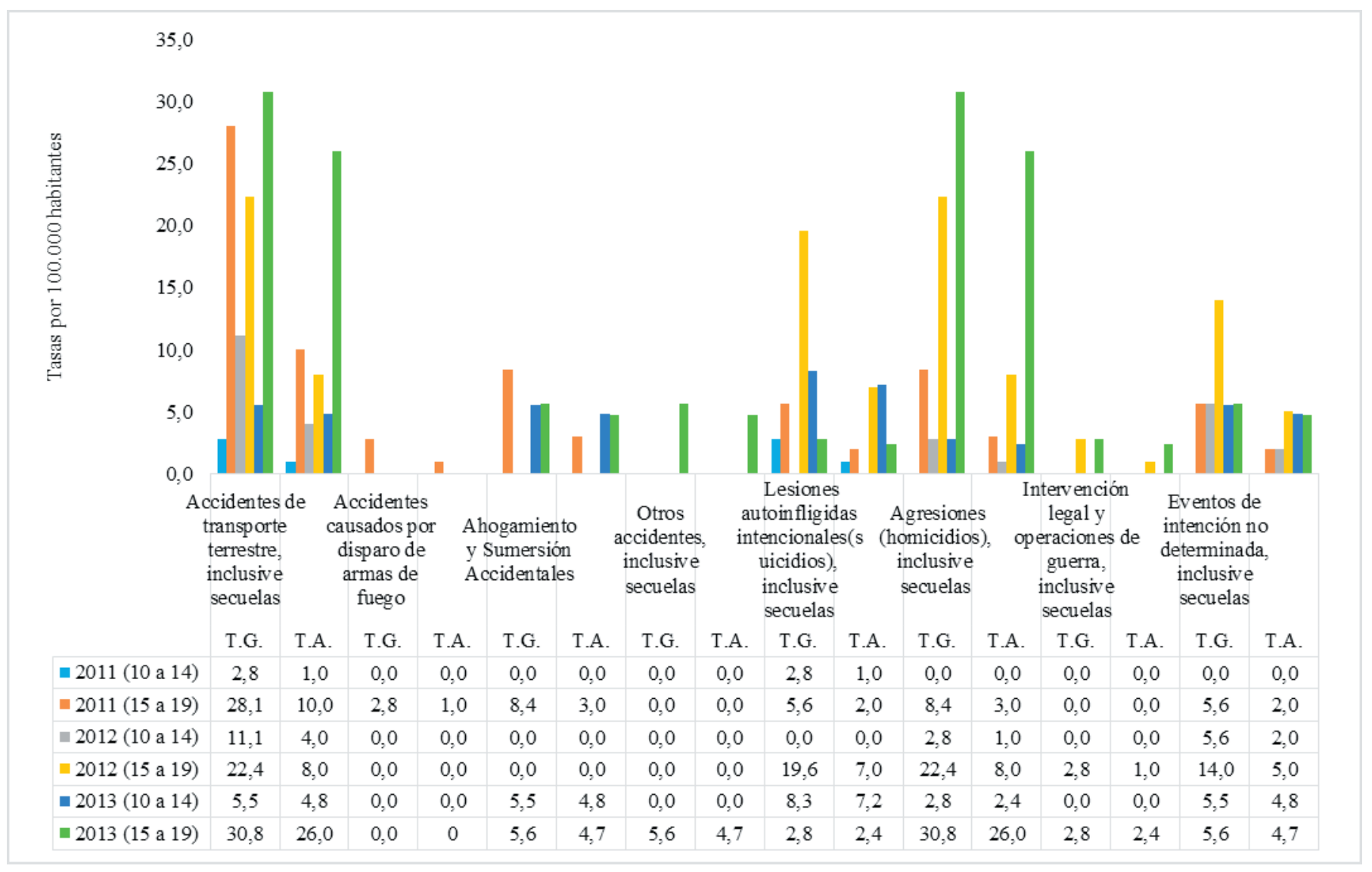

De los 19 municipios del departamento 17 (89,5\%) tuvieron defunciones, de ellos, Yopal 46 (46,5\%), Aguazul 12 (12,1\%), Paz de Ariporo 9 (9,1\%), Maní 5 (5,1\%), Támara, Tauramena y Trinidad 4 (4,0\%) respectivamente, Hato Corozal, La Salina, Nunchía, San Luis de Palenque y Villanueva 2 $(2,0 \%)$ cada uno; y, Orocué, Pore, Recetor y Sabanalarga $1(1,0 \%)$ respectivamente.

La tasa de mortalidad general por lesiones fatales en adolescentes fue de 138,2 por 100.000 habitantes, mientras que la tasa ajustada fue de 234,4 por 100.000 , en el grupo de 10 a 14 años de edad $(52,8 ; 45,4)$ y de $15-19$ (224,3; 189,9), según el sexo masculino $(220,0 ; 187,7)$ y femenino $(54,1 ; 46,2)$, (figura 1); distribuidas de acuerdo con el tipo de lesión, por accidentes de transporte terrestre 50,3; agresiones (homicidios) 33,5; lesiones autoinfligidas intencionales (suicidios) 19,5; eventos de intención no determinada 18,1; ahogamiento y sumersión accidentales 9,8; otros accidentes incluido secuelas e intervención legal y operaciones de guerra 2,8 respectivamente; y, accidentes causados por disparo de armas de fuego 1,4 (figuras 2 y 3 ).

Cuadro 2. Tasas específica y ajustada de mortalidad por 100.000 habitantes y años de vida potencial perdidos por municipio, sexo según lista $6 / 67$ CIE-10 OPS, lesiones fatales, adolescentes 10 a 14 años, Casanare, 2011-2013

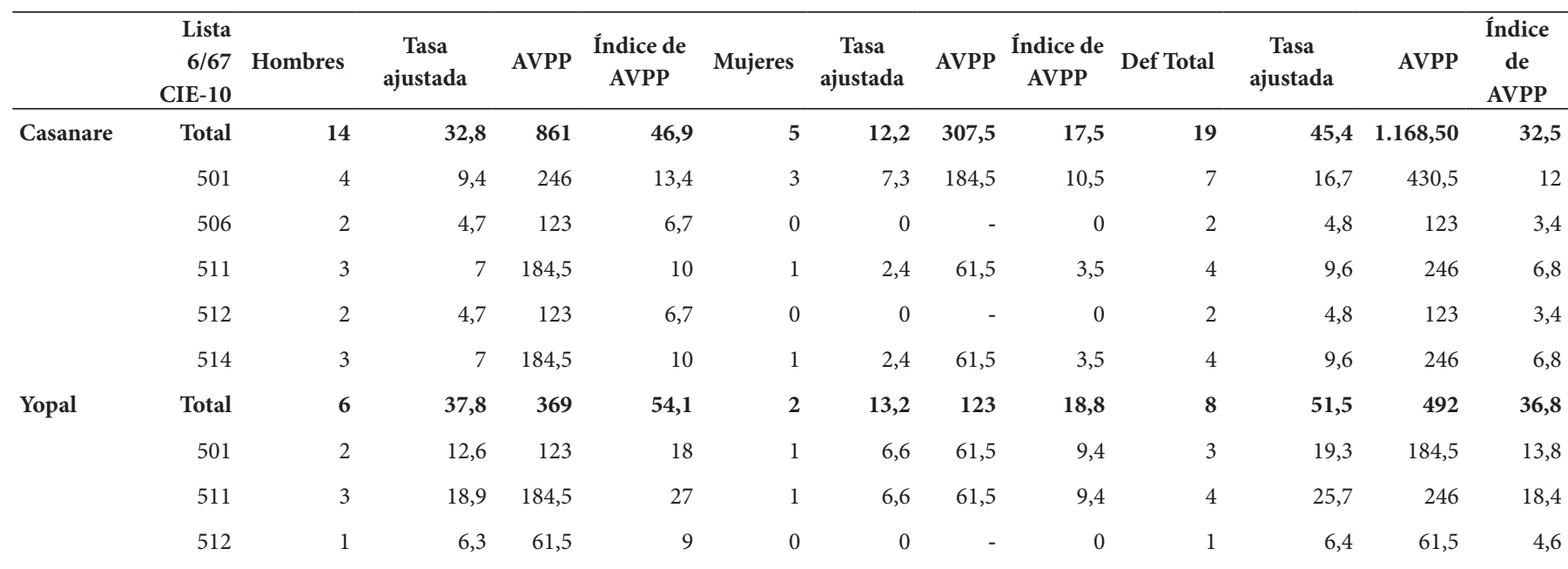




\begin{tabular}{|c|c|c|c|c|c|c|c|c|c|c|c|c|c|}
\hline \multirow[t]{3}{*}{ Aguazul } & Total & 3 & 65,9 & 184,5 & 94,3 & 0 & $\mathbf{0}$ & - & 0 & 3 & 67 & 184,5 & 47,9 \\
\hline & 501 & 1 & 22 & 61,5 & 31,4 & 0 & 0 & - & 0 & 1 & 22,3 & 61,5 & 16 \\
\hline & 506 & 2 & 43,9 & 123 & 62,9 & 0 & 0 & - & 0 & 2 & 44,7 & 123 & 32 \\
\hline $\begin{array}{l}\text { Hato } \\
\text { Corozal }\end{array}$ & 501 & 0 & 0 & - & 0 & 2 & 131,7 & 123 & 188,5 & 2 & 126,3 & 123 & 90,4 \\
\hline Maní & 514 & 1 & 70,8 & 61,5 & 101,3 & 0 & 0 & - & 0 & 1 & 74,8 & 61,5 & 53,5 \\
\hline \multirow[t]{2}{*}{$\begin{array}{l}\text { Paz de } \\
\text { Ariporo }\end{array}$} & Total & 1 & 28,1 & 61,5 & 40,3 & 1 & 29,6 & 61,5 & 42,4 & 2 & 57,7 & 123 & 41,3 \\
\hline & 514 & 1 & 28,1 & 61,5 & 40,3 & 1 & 29,6 & 61,5 & 42,4 & 2 & 57,7 & 123 & 41,3 \\
\hline Tauramena & 501 & 1 & 41,7 & 61,5 & 59,7 & 0 & 0 & - & 0 & 1 & 43 & 61,5 & 30,8 \\
\hline
\end{tabular}

Cuadro 3. Tasas específica y ajustada de mortalidad por 100.000 habitantes y años de vida potencial perdidos por municipio, sexo según lista 6/67 CIE-10 OPS, lesiones fatales, adolescentes 15 a 19 años, Casanare, 2011-2013

\begin{tabular}{|c|c|c|c|c|c|c|c|c|c|c|c|c|c|}
\hline & $\begin{array}{c}\text { Lista } \\
6 / 67 \\
\text { CIE-10 }\end{array}$ & Hombres & $\begin{array}{c}\text { Tasa } \\
\text { ajustada }\end{array}$ & AVPP & $\begin{array}{l}\text { Indice } \\
\text { de } \\
\text { AVPP }\end{array}$ & Mujeres & $\begin{array}{c}\text { Tasa } \\
\text { ajustada }\end{array}$ & AVPP & $\begin{array}{l}\text { Índice } \\
\text { de } \\
\text { AVPP }\end{array}$ & $\begin{array}{c}\text { Def } \\
\text { Total }\end{array}$ & $\begin{array}{c}\text { Tasa } \\
\text { ajustada }\end{array}$ & AVPP & $\begin{array}{l}\text { Índice } \\
\text { de } \\
\text { AVPP }\end{array}$ \\
\hline \multirow{7}{*}{ Casanare } & Total & 66 & 153,8 & $3.729,00$ & 205,3 & 14 & 33,9 & 791 & 45,2 & 80 & 189,9 & $4.520,00$ & 126,7 \\
\hline & 501 & 23 & 53,6 & $1.299,50$ & 71,5 & 6 & 14,5 & 339 & 19,4 & 29 & 68,8 & $1.638,50$ & 45,9 \\
\hline & 505 & 1 & 2,3 & 56,5 & 3,1 & 0 & 0 & - & 0 & 1 & 2,4 & 56,5 & 1,6 \\
\hline & 511 & 6 & 14 & 339 & 18,7 & 4 & 9,7 & 226 & 12,9 & 10 & 23,7 & 565 & 15,8 \\
\hline & 512 & 19 & 44,3 & $1.073,50$ & 59,1 & 3 & 7,3 & 169,5 & 9,7 & 22 & 52,2 & $1.243,00$ & 34,9 \\
\hline & 513 & 2 & 4,7 & 113 & 6,2 & 0 & 0 & - & 0 & 2 & 4,7 & 113 & 3,2 \\
\hline & 514 & 8 & 18,6 & 452 & 24,9 & 1 & 2,4 & 56,5 & 3,2 & 9 & 21,4 & 508,5 & 14,3 \\
\hline \multirow[t]{4}{*}{ Yopal } & 506 & 1 & 6,6 & 56,5 & 8,8 & 0 & 0 & - & 0 & 1 & 6,6 & 56,5 & 4,4 \\
\hline & 511 & 2 & 13,2 & 113 & 17,6 & 4 & 26,3 & 226 & 35,2 & 6 & 39,5 & 339 & 26,4 \\
\hline & 512 & 7 & 46,1 & 395,5 & 61,5 & 2 & 13,2 & 113 & 17,6 & 9 & 59,3 & 508,5 & 39,5 \\
\hline & 514 & 2 & 13,2 & 113 & 17,6 & 0 & 0 & - & 0 & 2 & 13,2 & 113 & 8,8 \\
\hline \multirow{4}{*}{ Aguazul } & Total & 7 & 151 & 395,5 & 201,5 & 2 & 43,6 & 113 & 58,2 & 9 & 195,2 & 508,5 & 130,3 \\
\hline & 501 & 2 & 43,1 & 113 & 57,6 & 2 & 43,6 & 113 & 58,2 & 4 & 86,8 & 226 & 57,9 \\
\hline & 506 & 3 & 64,7 & 169,5 & 86,3 & 0 & 0 & - & 0 & 3 & 65,1 & 169,5 & 43,4 \\
\hline & 512 & 2 & 43,1 & 113 & 57,6 & 0 & 0 & - & 0 & 2 & 43,4 & 113 & 29 \\
\hline
\end{tabular}




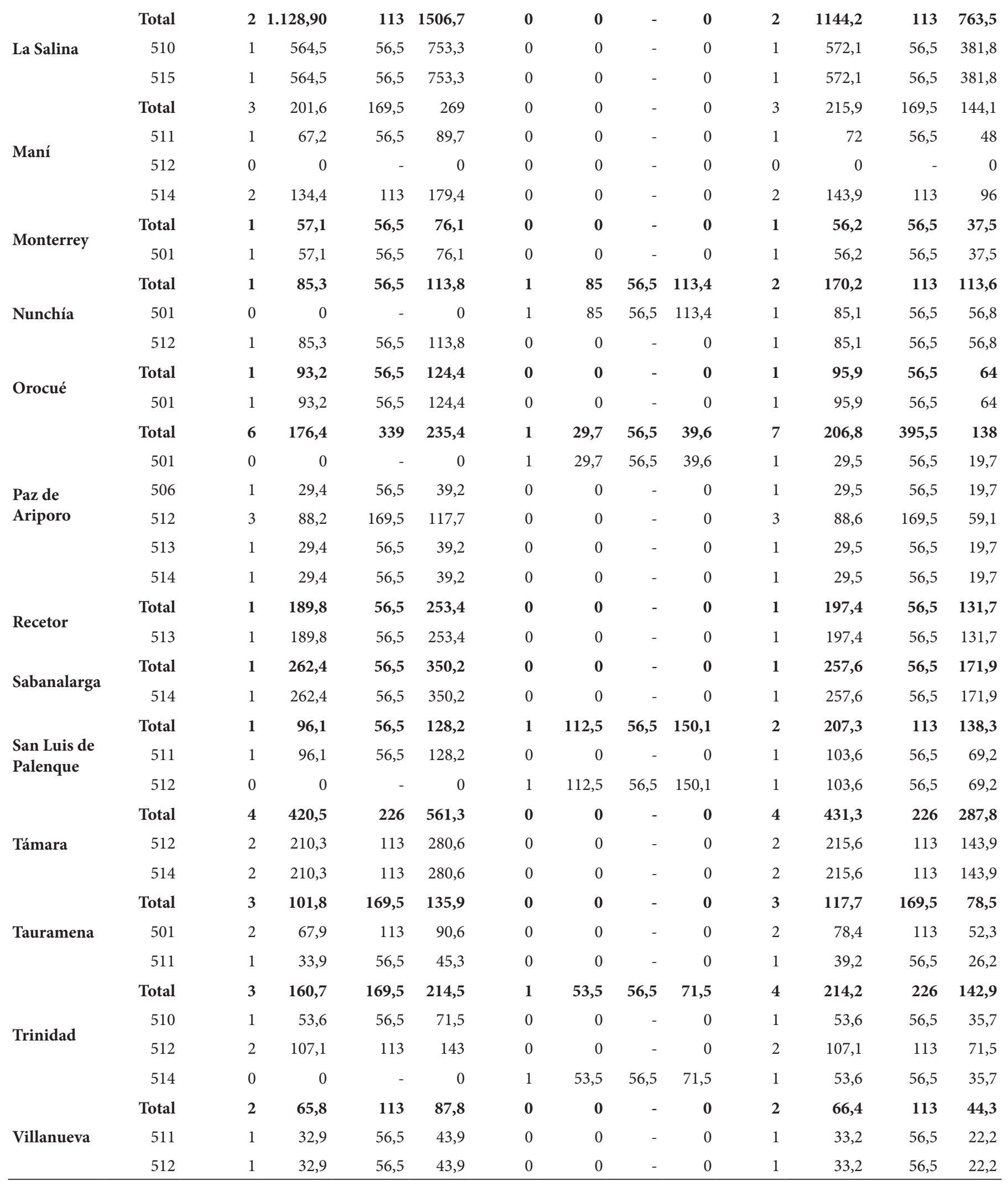


Cuadro 4. Tasas específica y ajustada de mortalidad por 100.000 habitantes y años de vida potencial perdidos por municipio, sexo según lista 6/67 CIE-10 OPS, lesiones fatales, adolescentes 10 a 19 años, Casanare, 2011-2013

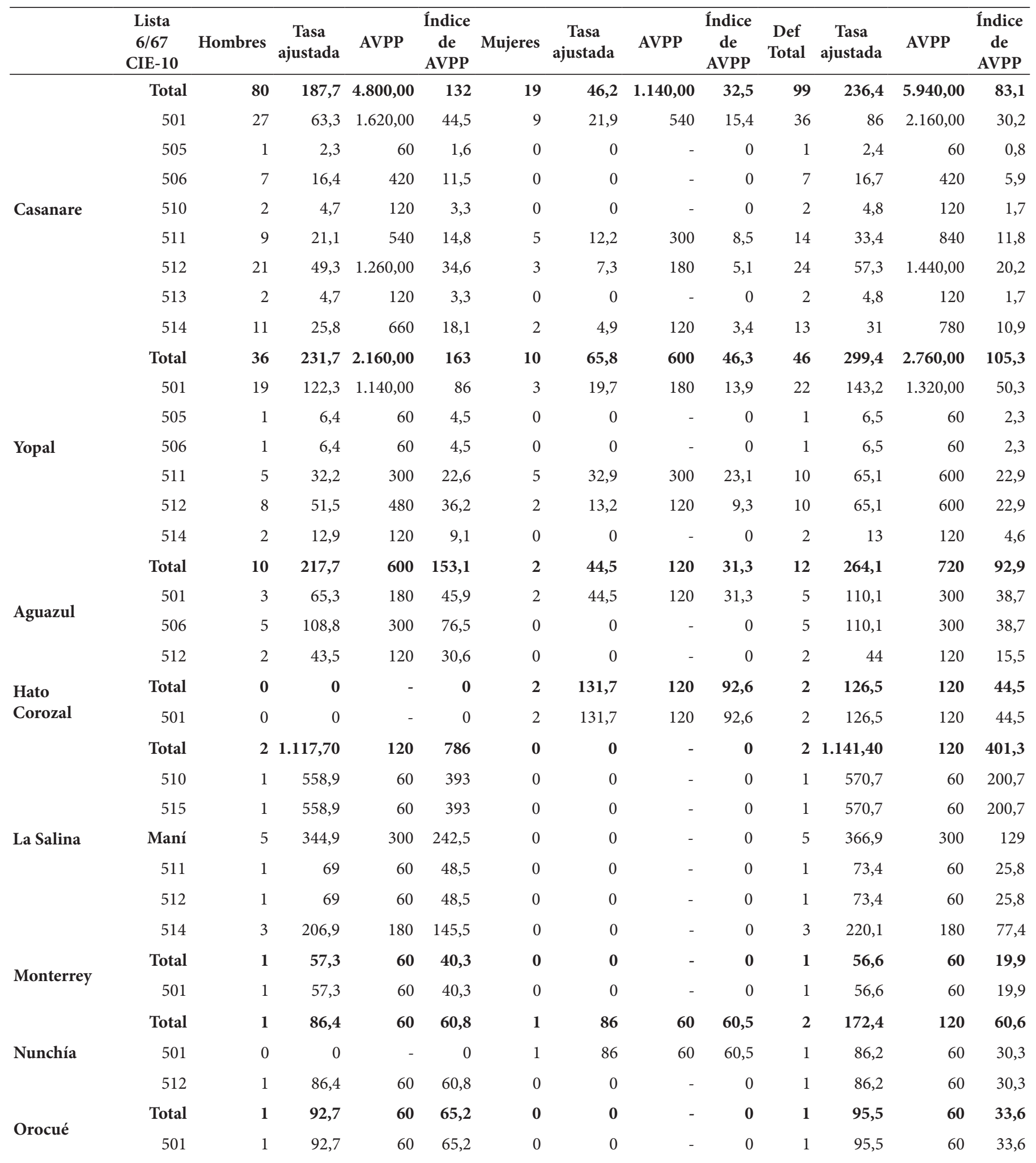




\begin{tabular}{|c|c|c|c|c|c|c|c|c|c|c|c|c|c|}
\hline & Total & 7 & 201,2 & 420 & 141,5 & 2 & 59,3 & 120 & 41,7 & 9 & 262,8 & 540 & 92,4 \\
\hline \multirow{5}{*}{$\begin{array}{l}\text { Paz de } \\
\text { Ariporo }\end{array}$} & 501 & 0 & 0 & - & 0 & 1 & 29,7 & 60 & 20,9 & 1 & 29,2 & 60 & 10,3 \\
\hline & 506 & 1 & 28,7 & 60 & 20,2 & 0 & 0 & - & 0 & 1 & 29,2 & 60 & 10,3 \\
\hline & 512 & 3 & 86,2 & 180 & 60,7 & 0 & 0 & - & 0 & 3 & 87,6 & 180 & 30,8 \\
\hline & 513 & 1 & 28,7 & 60 & 20,2 & 0 & 0 & - & 0 & 1 & 29,2 & 60 & 10,3 \\
\hline & 514 & 2 & 57,5 & 120 & 40,4 & 1 & 29,7 & 60 & 20,9 & 3 & 87,6 & 180 & 30,8 \\
\hline \multirow{2}{*}{ Pore } & Total & 1 & 92 & 60 & 64,7 & 0 & 0 & - & 0 & 1 & 95,8 & 60 & 33,7 \\
\hline & 514 & 1 & 92 & 60 & 64,7 & 0 & 0 & - & 0 & 1 & 95,8 & 60 & 33,7 \\
\hline \multirow{2}{*}{ Recetor } & Total & 1 & 196,3 & 60 & 138 & 0 & 0 & - & 0 & 1 & 201,6 & 60 & 70,9 \\
\hline & 513 & 1 & 196,3 & 60 & 138 & 0 & 0 & - & 0 & 1 & 201,6 & 60 & 70,9 \\
\hline \multirow[t]{2}{*}{ Sabanalarga } & Total & 1 & 263,6 & 60 & 185,4 & 0 & 0 & - & 0 & 1 & 259,7 & 60 & 91,3 \\
\hline & 514 & 1 & 263,6 & 60 & 185,4 & 0 & 0 & - & 0 & 1 & 259,7 & 60 & 91,3 \\
\hline \multirow{4}{*}{$\begin{array}{l}\text { San Luis de } \\
\text { Palenque }\end{array}$} & Total & 1 & 100,1 & 60 & 70,4 & 1 & 116,4 & 60 & 81,9 & 2 & 215,2 & 120 & 75,7 \\
\hline & 511 & 1 & 100,1 & 60 & 70,4 & 0 & 0 & - & 0 & 1 & 107,6 & 60 & 37,8 \\
\hline & 512 & 0 & 0 & - & 0 & 1 & 116,4 & 60 & 81,9 & 1 & 107,6 & 60 & 37,8 \\
\hline & Total & 4 & 435,5 & 240 & 306,3 & 0 & 0 & - & 0 & 4 & 446,6 & 240 & 157 \\
\hline \multirow[t]{3}{*}{ Támara } & 512 & 2 & 217,7 & 120 & 153,1 & 0 & 0 & - & 0 & 2 & 223,3 & 120 & 78,5 \\
\hline & 514 & 2 & 217,7 & 120 & 153,1 & 0 & 0 & - & 0 & 2 & 223,3 & 120 & 78,5 \\
\hline & Total & 4 & 149,9 & 240 & 105,4 & 0 & 0 & - & 0 & 4 & 164,2 & 240 & 57,7 \\
\hline \multirow[t]{3}{*}{ Tauramena } & 501 & 3 & 112,4 & 180 & 79 & 0 & 0 & - & 0 & 3 & 123,1 & 180 & 43,3 \\
\hline & 511 & 1 & 37,5 & 60 & 26,3 & 0 & 0 & - & 0 & 1 & 41 & 60 & 14,4 \\
\hline & Total & 3 & 161,8 & 180 & 113,8 & 1 & 53,3 & 60 & 37,5 & 4 & 214,4 & 240 & 75,4 \\
\hline \multirow{4}{*}{ Trinidad } & 510 & 1 & 53,9 & 60 & 37,9 & 0 & 0 & - & 0 & 1 & 53,6 & 60 & 18,8 \\
\hline & 512 & 2 & 107,9 & 120 & 75,9 & 0 & 0 & - & 0 & 2 & 107,2 & 120 & 37,7 \\
\hline & 514 & 0 & 0 & - & 0 & 1 & 53,3 & 60 & 37,5 & 1 & 53,6 & 60 & 18,8 \\
\hline & Total & 2 & 66,7 & 120 & 46,9 & 0 & 0 & - & 0 & 2 & 67,4 & 120 & 23,7 \\
\hline \multirow[t]{2}{*}{ Villanueva } & 511 & 1 & 33,3 & 60 & 23,4 & 0 & 0 & - & 0 & 1 & 33,7 & 60 & 11,9 \\
\hline & 512 & 1 & 33,3 & 60 & 23,4 & 0 & 0 & - & 0 & 1 & 33,7 & 60 & 11,9 \\
\hline
\end{tabular}

Las tasas específica y ajustada de mortalidad, los AVPP y el índice de AVPP por municipio, grupo etario, sexo y tipo de lesiones fatales según la lista 6/67 CIE-10 de OPS se presenta en las tablas 2-4. El índice de AVPP por lesiones fatales en el departamento de Casanare entre los adolescentes de 10 a 19 años de edad indica que se pierden 83,1 años por cada 1.000 habitantes.

\section{Discusión}

Durante el período a estudio, en Casanare, se observa un ascenso en el número total de muertes en adolescentes entre cada uno de los años a estudio, con un incremento de 73,9\% al comparar 2011 respecto de 2013; y, concentrados en el grupo etario de 15 a 19 años del sexo masculino, por cada cuatro adolescentes varones fallece una adolescente. En relación con el sexo de las víctimas, los más afectados son los adolescentes del sexo masculino, por todas las causas de lesiones fatales registradas en el departamento, situación que coincide con la reportada en el Informe mundial de sobre prevención de las lesiones en los niños de 2012, según el cual, en la región de las Américas, el riesgo de morir por lesiones de causa externa es de 1,5 a 2,7 veces mayor en los menores de 18 años del sexo masculino (6). En cuanto a la distribución del número de casos por municipio, llama la atención que en los municipios de Chámeza y Sácama no se presentaron defunciones en adolescentes durante el período a estudio, mientras que más de la mitad se concentró en los municipios de Yopal, Aguazul, Paz de Ariporo y Maní; y en los municipios de Monterrey, Orocué, Pore, Recetor y Sabanalarga solo se presentó un fallecimiento.

En este estudio de acuerdo con la lista 6/67 CIE-10 de la OPS, no se presentaron lesiones fatales por caídas; accidentes causados por máquinas y por instrumentos cortantes o punzantes; exposición al humo, fuego y llamas; y, complicaciones de la atención médica y quirúrgica. La primera causa de mortalidad por lesiones fatales fue por accidentes de transporte seguido por homicidios y suicidio, situación diferente a la encontrada en otras ciudades del país como Medellín en la cual los homicidios se encontraron en el primer lugar seguido de los accidentes de transporte, para el período 1999-2006 (27), así como a las reportadas por el ICML-CF para el país, que en 2011, 2012 y 2013 ocuparon el primer lugar, seguido por 
accidentes de transporte, pero las defunciones por suicidio ocuparon el quinto lugar $(10,18,28)$. La tasa de mortalidad por accidentes de transporte fue superior a la reportada en el Informe mundial sobre seguridad vial para los países de ingresos medios de 20,1 por 100.000 , en comparación con 8,7 en los de ingresos elevados y 18,3 en los de ingresos bajos $(29,30)$. Se ha estimado que por cada joven que muere por accidente hay 15 heridos graves y 30 o 40 heridos leves (31)

Así mismo, existen estudios que demuestran que la distribución de los accidentes de tránsito es generalmente influenciada por factores socioeconómicos (32); las personas más pobres representan la mayoría de las víctimas quienes carecen de apoyo permanente en caso de lesiones de larga duración; así como, también tienen un acceso limitado a la atención de urgencia después de un choque. Además, los costos de la atención médica prolongada, la desaparición de las personas que sustentan a la familia, el costo de un funeral o la pérdida de ingresos por discapacidad pueden sumir a las familias en la pobreza (33); en este sentido, se relaciona el índice de Necesidades Básicas Insatisfechas (NBI) como uno de los criterios para medir el nivel y la intensidad de la pobreza (34) según el cual el 35,5\% de las personas en Casanare tienen NBI y a su vez el $13,6 \%$ vive en la miseria; es decir que los niveles de pobreza y desigualdad en el departamento podrían tener relación con el elevado índice de lesiones fatales por accidentes de transporte.

Por otra parte, los eventos de intención no determinada, representan un alto porcentaje dentro de las lesiones fatales por causa externa en Casanare, principalmente en el grupo de adolescencia tardía (13\%) situación muy diferente a ciudades como Medellín en la cual no supera el $1 \%$. Este grupo de eventos de intención no determinada podrían distorsionar el análisis de la mortalidad puesto que incide en la calidad de la información ya que los datos suministrados por el informante son insuficientes para distinguir la intencionalidad de los mismos (35).

El suicidio fue la tercera causa externa de muerte en los adolescentes de Casanare ascendiendo del 2011 al 2012 y con descenso en 2013, con mayor prevalencia en los jóvenes entre 15 a 19 años. La tasa fue mayor en el género masculino ( 8,2 casos por 100.000 habitantes), la OMS describe para 47 países miembros, mayores tasas en mujeres que en hombres lo cual contrasta con lo encontrado en este estudio para el grupo de 10 a 19 años (36).

La problemática del homicidio va en incremento en la región de las Américas la cual ocupa el segundo lugar a nivel mundial, solamente superada por África (37); en Casanare ocupó el cuarto lugar dentro de las lesiones fatales por causa externa con mayor tasa en el género masculino. Las muertes por homicidios reflejan apenas una parte del problema de la violencia $(24,2 \%)$ en Casanare y se ha considerado tradicionalmente como indicador de violencia, resaltando que Colombia aún vive el mayor conflicto militar sin resolver de las Américas y se destaca por tener las mayores tasas de mortalidad por homicidios y muertes por conflicto armado de la región generando impacto negativo en la productividad, menor crecimiento económico y mayores niveles de pobreza que a su vez alimentan los factores de riesgo estructurales para una mayor violencia en el futuro $(38,39)$.

Las lesiones fatales de causa externa son responsables de un gran número de AVPP en Casanare dentro de los cuales se destacan los accidentes de transporte terrestre con el 36,2\% del total de AVPP, homicidios (23,9\% del total de AVPP) y suicidios (14,2\% del total de AVPP) afectando principalmente a hombres. Resultados similares al estudio realizado en Cali, en el cual se resalta la magnitud de AVPP por causas externas, sobre todo por homicidios, suicidios y accidentes de tránsito y dentro del cual los hombres jóvenes sufrieron una pérdida casi nueve veces mayor que la que se observó en las mujeres jóvenes (40). El índice de AVPP por accidentes de transporte terrestre fue de 30,2 años perdidos por cada 1.000 habitantes, 23 veces superior al reportado por el país en 2010, año en el cual se perdieron 7,14 años por cada 1.000 habitantes (41); por municipio el mayor índice lo reporta La Salina, seguido de Yopal.

La disminución en los índices de mortalidad por causa externa en Casanare representa un desafío multi e intersectorial pues no solamente se enfrenta un problema en salud pública sino aquellos generados por los costos sociales de estos eventos, más aún cuando en muchos casos es producto de la violencia y la intolerancia social, aunado al hecho que las lesiones son eventos prevenibles cuyos factores de riesgo deben ser identificados e intervenidos en su gran mayoría (42); por tanto, es necesario congregar esfuerzos desde todos los sectores para reducir la mortalidad de todos los niños y reducir los niveles de pobreza (29).

Las fuentes de información utilizadas para estadísticas vitales se constituyen en una de las principales herramientas que aportan a la caracterización de esos eventos, por tanto, es importante fortalecer las instituciones que integran este sistema de manera que se mejore la calidad de la información para contar con información más consistente y veraz que permita determinar la dinámica del problema en cada municipio así como las posibles causas orientados a encontrar soluciones frente a los factores relacionados con la mortalidad por estas causas.

Es indispensable la asignación de recursos financieros y humanos para contrarrestar la mortalidad ocasionada por accidentes de tránsito, de manera que no sólo se haga más segura la infraestructura vial sino que creen y apliquen políticas para el cumplimiento de las normas de tránsito en la población, puesto que la voluntad y el compromiso político son esenciales para salvar vidas y prevenir la discapacidad por esta causa. Entre las intervenciones que propone la OMS cabe citar la aplicación de legislación para controlar la velocidad y el consumo de alcohol, el uso obligatorio de cinturones de seguridad y cascos, y un diseño y un uso más seguros de la vía pública (29).

La problemática de suicidios y homicidios se debe abordar desde una perspectiva de prevención primaria con medidas orientadas a reducir los factores de riesgo de violencia en los individuos, las relaciones, las comunidades y la sociedad de manera integral. Según recomendaciones de la OPS algunas de estas actividades deben encaminarse a educar, brindar opciones de vida, promover el desarrollo personal y social, concientizar sobre la importancia de las buenas relaciones sociales, fortalecer los lazos familiares y vínculos afectivos, asesorar a las potenciales víctimas y victimarios y mejorar el entorno físico y socioeconómico (43).

\section{Conflicto de interés}

Los autores declaran que no hay conflicto de intereses. 


\section{Agradecimientos}

Estudio financiado con capacidades y recursos propios de la Secretaría de Salud de Casanare.

Todos los autores participaron en el diseño, análisis e interpretación de los datos, escritura y corrección del manuscrito y aprobación del texto final y declaran no tener conflictos de interés

Fecha de envío: 24 de noviembre de 2015

\section{Referencias}

1. Espitia-Hederman V, Paulozzi L. Manual de capacitación en la vigilancia de las lesiones. Guía para el Instructor. Atlanta: Centros para el Control y la Prevención de Enfermedades; Centro Nacional para la Prevención y el Control de las Lesiones; 2005. $187 \mathrm{p}$.

2. Heredia D, Moreno SL. Comportamiento de las lesiones de causa externa. Colombia, 2014. En: Instituto Nacional de Medicina Legal y Ciencias Forenses., Grupo Centro de Referencia Nacional sobre Violencia., editors. Forensis Datos para la vida 2014. 1 ed. Bogotá, D. C: Imprenta Nacional; 2015. p. 19-90.

3. World Health Organization. Injuries and violence: the facts. Geneva: WHO Press; 2010. 19 p.

4. Bartalomeos K, Kipsaina C, Grills N, Ozanne-Smith J, Peden M. Fatal injury surveillance in mortuaries and hospitals: a manual for practitioners. Geneve: World Health Organization; 2012. 92 p.

5. Pinheiro PS. Acabar con la violencia contra los niños, niñas y adolescentes. En: Pinheiro PS, editor. Informe mundial sobre la violencia contra los niños y niñas: Estudio del Secretario General de las Naciones Unidas sobre la Violencia contra los niños; 2006. p. 3-27.

6. Peden M, Oyegbite K, Ozanne-Smith J, Hyder AA, Branche C, Rahman AF, et al. Informe mundial sobre prevención de las lesiones en los niños. Washington, D. C.: Organización Mundial de la Salud. Organización Panamericana de la Salud. UNICEF; 2012. 40 p.

7. Ministerio de Salud y Protección Social. Análisis de Situación de Salud - Colombia, 2014. Bogotá. D. C.: Imprenta Nacional de Colombia; 2015. 170 p.

8. Arias LF. Mujeres indígenas, víctimas invisibles del conflicto armado en Colombia. Forensis 2012. Datos para la vida Herramienta para la interpretación, intervención y prevención de lesiones de causa externa en Colombia. Bogotá, D.C.: Instituto Nacional de Medicina Legal y Ciencias Forenses; 2013. p. 281321.

9. Insuasty JR. Violencia interpersonal. Forensis 2012. Datos para la vida Herramientas para la interpretación, intervención y prevención de lesiones de causa externa en Colombia. Bogotá, D.C.: Instituto Nacional de Medicina Legal y Ciencias Forenses; 2013. p. 157-96.

10. Moreno SL. Lesiones de causa externa. Colombia, 2012. En: Instituto Nacional de Medicina Legal y Ciencias Forenses, Grupo Centro de Referencia Nacional sobre Violencia., editores. Forensis 2012. Datos para la vida Herramientas para la interpretación, intervención y prevención de lesiones de causa externa en Colombia. 1a ed. Bogotá. D.C.: Instituto Nacional de Medicina Legal y Ciencias Forenses; 2013. p. 11-105.

11. Moreno SL. Muertes y lesiones por accidentes de transporte, Colombia, 2012. Forensis 2012. Datos para la vida Herramienta para la interpretación, intervención y prevención de lesiones de causa externa en Colombia. Bogotá, D.C.: Instituto Nacional de Medicina Legal y Ciencias Forenses; 2013. p. 351-402.
12. Muñoz O. Indicadores de infancia y adolescencia. Yopal: Secretaría de Salud Departamental de Casanare; 2011. 83 p.

13. Rodríguez JM. Muertes y lesiones accidentales en Colombia, 2012. Forensis 2012. Datos para la vida Herramientas para la interpretación, intervención y prevención de lesiones de causa externa en Colombia. Bogotá, D.C.: Instituto Nacional de Medicina Legal y Ciencias Forenses; 2013. p. 405-51.

14. Velasco VL, Lozano N. Comportamiento de la violencia intrafamiliar, Colombia, 2012. Forensis 2012. Datos para la vida Herramienta para la interpretación, intervención y prevención de lesiones de causa externa en Colombia. Bogotá, D. C.: Instituto Nacional de Medicina Legal y Ciencias Forenses; 2013. p. 199277.

15. Rodríguez-García J. Descripción de la mortalidad por departamentos. Colombia año 2000. Documento de trabajo ASS/ DT 016-05. Bogotá: CENDEX; 2005.

16. Secretaría de Salud de Casanare, Red Salud Casanare E.S.E., Universidad del Rosario. Plan departamental de salud mental, Casanare, 2010-2013. Yopal, Casanare: La Secretaría; 2010. 135 p.

17. Instituto Nacional de Salud, Observatorio Nacional de Salud. Segundo informe ONS: mortalidad 1998-2011 y situación de salud en los municipios de frontera terrestre en Colombia. Bogotá, D.C.: Imprenta Nacional de Colombia; 2013. 237 p.

18. Moya DM. Comportamiento de las lesiones de causa externa, Colombia, 2013. Forensis 2013 Datos para la vida Herramienta para la interpretación y prevención de lesiones de causa externa en Colombia. Bogotá, D.C.: Instituto de Medicina Legal y Ciencias Forenses; 2014. p. 19-76.

19. Blanco M. El enfoque del curso de vida: orígenes y desarrollo. Revista Larinoamericana de Población. 2011;5(8):5-31.

20. Ministerio de Salud y Protección Social. Enfoque de Curso de Vida: Minsalud; 2015 [cited 10 de octubre de 2014]. Available from: https://www.minsalud.gov.co/sites/rid/Lists/ BibliotecaDigital/RIDE/VS/PP/ABCenfoqueCV.pdf.

21. Organización Panamericana de la Salud. Clasificación Internacional de Enfermedades: Preparación de Listas Cortas para la Tabulación de Datos. Boletín Epidemiológico / OPS. 2002;23(4):2-6.

22. Organización Panamericana de la Salud, Organización Mundial de la Salud. Clasificación Estadística Internacional de Enfermedades y Problemas Realacionados con la Salud. Décima Revisión. Publicación Científica No. 554. 2008 ed. Washington, D.C.: Organización Panamericana de la Salud. Oficina Sanitaria Panamericana, Oficina Regional de la Organización Mundial de la Salud; 1995. 758 p.

23. Fondo de las Naciones Unidas para la Infancia - UNICEF. Estado mundial de la infancia 2011. La adolescencia una época de oportunidades. Nueva York: UNICEF; 2011. 138 p.

24. Instituto Mexicano de Seguro Social. Población estándar mundial 2016 [cited 11 de febrero de 2016]. Available from: http://datos. imss.gob.mx/dataset/enfermedades-cr\%C3\%B3nicas/resource/ ae9ed6bc-058c-4556-bb50-a78c808bcc0c\#\{view-graph:\{graphO ptions:\{hooks:\{processOffset:\{\},bindEvents: \{\}$\}\}\}$, graphOptions: \{ hooks:\{processOffset:\{\},bindEvents: \{\}$\}\}\}$.

25. República de Colombia, Ministerio de Salud. Resolución por la cual se establecen las normas científicas, técnicas y administrativas para la investigación en salud. Resolución 008430 de 1993 (octubre 4) 1993.

26. República de Colombia, Ministerio de la Protección Social. Decreto por el cual se crea y reglamenta el Sistema de Vigilancia en Salud Pública y se dictan otras disposiciones. Decreto 3518 de 2006 (octubre 09). 
27. Cardona D. Mortalidad por causas externas en Medellín, 19992006. Papeles de Población. 2008;14(56):233-56.

28. Moreno SL, Carreño PA. Lesiones de causa externa, Colombia, 2011. Forensis 2011. Datos para la vida Herramienta para la interpretación, intervención y prevención de lesiones de causa externa en Colombia: Instituo Nacional de Medicina Legal y Ciencias Forenses; 2012. p. 11-63.

29. Organización Mundial de la Salud, Banco Mundial. Informe mundial sobre prevención de los traumatismos causados por el tránsito: resumen. Ginebra: OMS, 2004.

30. Organización Mundial de la Salud, Departamento de Prevención de la Violencia y los Traumatismos y Discapacidad (VIP). Informe sobre la situación mundial de la seguridad vial 2013. Apoyo al decenio de acción. Resumen. Ginebra: OMS; 2013. 11 p.

31. Organización Panamericana de la Salud. Adolescencia y juventud: agunos aspectos demográficos y epidemiológicos. Boletín Epidemiológico / OPS. 1985;6(6):1-7.

32. Nantulya VM, Reich MR. Equity dimensions of road traffic injuries in low-and middle-income countries. Injury control and safety promotion. 2003;10(1-2):13-20.

33. Hijar M, Vazquez-Vela E, Arreola-Risa C. Pedestrian traffic injuries in Mexico: a country update. Injury control and safety promotion. 2003;10(1-2):37-43.

34. Pérez GJ. Dimensión espacial de la pobreza en Colombia. Documentos de trabajo sobre economía regional. 2005(54).

35. Cardona D, Peláez E, Aidar T, Ribotta B, Alvarez MF. Mortalidad por causas externas em tres ciudades latinoamericanas: Córdoba (Argentina), Campinas (Brasil) y Medellín (Colombia), 19802005. R Bras Est Pop. 2008;25(2):335-52.

36. Cendales R, Vanegas C, Fierro M, Córdoba R, Olarte A. Tendencias del suicidio en Colombia, 1985-2002. Rev Panam Salud Pública. 2007;22(4):231-8.

37. Organización Panamericana de la Ssalud, Organización Mundial de la Salud. Informe mundial sobre la violencia y la salud: resumen. Washington,D.C.2002.

38. Buvinic M, Morrison A, Orlando MB. Violencia, crimen y desarrollo social en América Latina y el Caribe: Universidad Autónoma del Estado de México; 2005.

39. Moreno C, Cendales R. Mortalidad y años potenciales de vida perdidos por homicidios en Colombia. Rev Panam Salud Publica. 2011;30(4):342-53.

40. Grisales Hugo, Caicedo Beatriz, Serna Catalina, Uribe Diana. Causas de mortalidad en jóvenes y su contribución al cambio en la esperanza de vida: Cali, 1989-1999. Colombia Médica. 2005;36(2):85-93.

41. León YL, S;anchez MA. Años potencialmente perdidos por accidentes de tránsito, Colombia 2010. Revista CES Salud Pública. 2011;2(2):159-68.

42. Rodríguez-Hernández JM. Accidentes y lesiones: necesidad de cambio de paradigma. Rev Salud Publica (Bogota). 2015;17(3):321-22.

43. Krug EG, Dahlberg LL, Mercy AB, Lozano R. Informe mundial sobre la violencia y la salud. Washington, D. C.: Organización Panamericana de la Salud, Oficina Sanitaria Panamericana, Oficina Regional de la Organización Mundial de la Salud; 2003. $374 \mathrm{p}$. 